\title{
The Application of Hybrid Genetic Algorithms Model in Solving Product Optimization Problem
}

\author{
Shao Xiufeng \\ Department of Information \\ Beijing City University \\ Beijing, China \\ shaoxiufeng@bcu.edu.cn \\ $\mathrm{Li} \mathrm{Li}$ \\ Department of Information \\ Beijing City University \\ Beijing, China \\ lily@bcu.edu.cn
}

\author{
Zhang Jian \\ IT Application Department \\ China Electric Power Research Institute \\ Beijing, China \\ zjsxf@epri.sgcc.com.cn
}

\begin{abstract}
We introduce basic theory of genetic algorithms in this paper, Build one hybrid genetic algorithms model base on genetic algorithms in solving product optimization problem. And we realize the model by software. The disposal result of real data tells us that the model has good practicability and accuracy to the problem of product optimization problem.
\end{abstract}

Keywords-genetic algorithms; hybrid genetic algorithms; product optimization; fitness function

\section{INTRODUCTION}

The final aim of every manufacturer is to realize the maximal benefit how to use resource fully under the condition of limit resource. We can solve this problem by genetic or developmental algorithms, but there is bug if we use single algorithms. We combine the two algorithms to build hybrid genetic algorithms in this paper, and solve the product optimization problem very good.

\section{GENETIC ALGORITHMS DESCRIPTION}

\section{A. Brief of genetic algorithms}

The genetic algorithms are one self-fit global probability search algorithms that genetic and evolution procedure of biology is simulated in the environment. It is provided earliest by Holland professor of university of American Mizhian, and origin from the research of nature and manpower self-fit system in 1960 years. ${ }^{[1]}$ De Jong carry out a lot pure number function optimize compute experiment base on genetic algorithms in 1970 years. ${ }^{\text {[2] }}$ Goldberg form basic framework by sum up and base on a serial research work in 1980 years. ${ }^{[3]}$

\section{B. Basic term of genetic algorithms}

The genetic algorithms are one new search and optimize algorithms that simulate genetic choice and natural eliminate through selection or contest's biology evolutionism base on Darwin's theory. The theory of Darwin thinks that every species more and more fit environment during the development procedure. Every basic character is inherited by next era, but net era is not equal to father era. The character is saved if it fits environment, and this is the theory of survival of the fittest. The genetic theory of Mendel thinks that genetic code encapsulation in every cell, and is included in the chromosome by the form of gene. The position of every gene controls has special place and control some special property, the position of gene named gene seat in the chromosome. Every unit has definite fit to the environment. The gene across and variation can produce next era better. The fit of species to the environment continues improve in nature by nature choice. We can get conclusion that breed of biology provides the chance to appear good character, nature choice saves the good character. The species is better by this method. The idiographic method is copy, across and variation. The genetic algorithms realize optimization procedure by simulating the three methods.

The genetic algorithms express the solution of the problem as chromosome. We build chromosome group by many feasible solutions. We put them in the condition of problem environment, choose chromosome of fit environment to copy according to the rule of survival of the fittest, produce new era chromosome colony by across and variation operator. We get optimization by converge the unit of fittest environment after continue evolution of every era. The operation of genetic algorithms is going on in the problem environment, the environment is fit function.

\section{Evaluation step of genetic algorithms}

The three basic terms of genetic algorithms, code, genetic operator and fit function. 
(1)Code. Among genetic algorithms, we first should change research space into expression space, which operation is called code. The adverse operation is called decode. The spaces of expression space are called individual or chromosome. Individual usually is expressed by string, each bit of string is called gene. Many individuals constitute a population to be used by genetic algorithms.

(2)Genetic operator. Superior wined and inferior washed out process is called genetic operator during evolution. The genetic algorithms mainly include three genetic operations: copy, across and variation.

(i)Copy is that forming new generation by exchanging and variation from father's good individuals with high fit.

(ii)Across is a genetic exchange process between two chromosomes.

(iii) Variation is gene's random change process of a chromosome.

The process of (ii) and (iii) can produce ideal chromosomes.

(3)Fit function. Among genetic algorithms, define a match function to measure a chromosome's extend. This is fit function. It is not confined by continuum and differentiability.

\section{Evaluation step of genetic algorithms}

We express the problem into chromosome first and build chromosome group. We put them in the condition, choose chromosome of fit environment to copy according to the rule of survival of the fittest, and produce new era chromosome colony by across and variation operator. We get optimization by converge the unit of fittest environment after continue evolution of every era.

The four ready works must be finished first if we want to use genetic algorithms to solve the problem.

(1)We ensure express scheme, that is how to express every point of problem space into binary string of sure length.

(2)We ensure the measurement if fit, that is how to compute fit value of every sure length character string.

(3)We ensure parameter and variable, that is scale $\mathrm{M}$ of colony, max era $\mathrm{N}$, copy probability $P_{r}$, across probability $P_{c}$,variation probability $P_{m}$ and so on.

(4)We ensure the method of produce the result and the rule of stop run.

We can execute genetic algorithms after finishing ready work. The main step of genetic algorithms as follows.

(1)We produce initial colony random by one character string group that confirm length.

(2)We execute step (i) and (ii) until satisfy stop rule.

(i)Compute fit value of every unit in the colony

(ii)Produce next era colony by copy, across and variation operator

(3)We name the execute result of genetic algorithms that two best unit strings of every era. The result can be expressed one solution (approximate solution).
The basic genetic algorithms as follows picture 1 . Variable $\mathrm{k}$ is current era number, and $\mathrm{m}$ is colony scale.

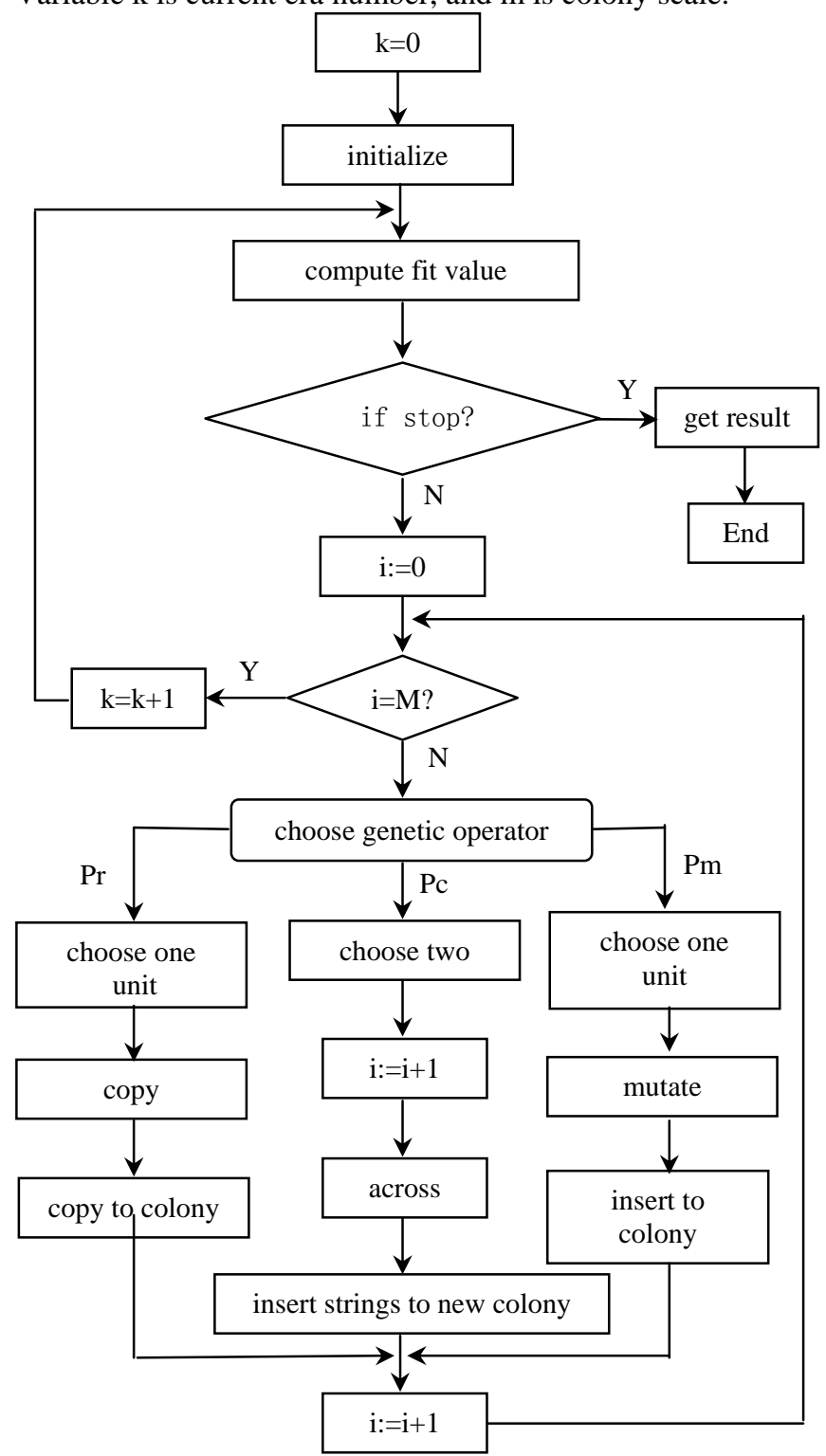

Figure 1 basic genetic algorithms framework

\section{ApPlicAtion In SOlving PRODUCt OptimizATION PROBLEM}

\section{A. Bring forward problem}

Some factory can produce fifty products, and the material of every product as table 1 . The unit of material is $\mathrm{M}$, the unit of profit is YUAN.

Table 1 product use material and profit

\begin{tabular}{|c|c|c|c|c|c|c|c|c|}
\hline product & $\mathbf{1}$ & $\mathbf{2}$ & $\mathbf{3}$ & $\mathbf{4}$ & $\mathbf{5}$ & $\mathbf{6}$ & $\mathbf{7}$ & $\mathbf{8}$ \\
\hline material & 253 & 245 & 243 & 239 & 238 & 237 & 236 & 235 \\
\hline profit & 200 & 200 & 240 & 240 & 225 & 225 & 235 & 235 \\
\hline
\end{tabular}




\begin{tabular}{|c|c|c|c|c|c|c|c|c|}
\hline product & 9 & 10 & 11 & 12 & 13 & 14 & 15 & 16 \\
\hline material & 234 & 232 & 231 & 230 & 229 & 228 & 227 & 226 \\
\hline profit & 110 & 140 & 160 & 180 & 150 & 130 & 170 & 180 \\
\hline product & 17 & 18 & 19 & 20 & 21 & 22 & 23 & 24 \\
\hline material & 224 & 217 & 213 & 207 & 203 & 201 & 195 & 194 \\
\hline profit & 190 & 230 & 220 & 280 & 210 & 205 & 195 & 190 \\
\hline product & 25 & 26 & 27 & 28 & 29 & 30 & 31 & 32 \\
\hline material & 191 & 188 & 187 & 177 & 175 & 171 & 169 & 168 \\
\hline profit & 180 & 170 & 160 & 140 & 175 & 185 & 160 & 160 \\
\hline product & 33 & 34 & 35 & 36 & 37 & 38 & 39 & 40 \\
\hline material & 166 & 164 & 161 & 160 & 158 & 150 & 149 & 147 \\
\hline profit & 165 & 170 & 190 & 160 & 140 & 150 & 130 & 120 \\
\hline product & 41 & 42 & 43 & 44 & 45 & 46 & 47 & 48 \\
\hline material & 141 & 140 & 139 & 136 & 135 & 132 & 128 & 126 \\
\hline profit & 110 & 140 & 135 & 145 & 155 & 160 & 165 & 125 \\
\hline product & 49 & 50 & & & & & & \\
\hline material & 122 & 120 & & & & & & \\
\hline profit & 125 & 120 & & & & & & \\
\hline
\end{tabular}

The total number of material is five thousands meter. The request of manufacturer is to realize the maximal benefit how to use resource fully under the condition of limit resource.

\section{B. Abstract math model}

We can abstract the problem into bag problem wide, math model as follows.

$$
\left\{\begin{array}{l}
\max \sum_{i=1}^{50} P_{i} X_{i} \\
\text { s.t. } \sum_{i=1}^{50} R_{i} X_{i} \leq 5000 \\
X_{i} \in\{0,1\}, 1 \leq i \leq n .
\end{array}\right.
$$

thereinto, $\mathrm{P}=\left(\mathrm{P}_{1}, \mathrm{P}_{2}, \cdots, \mathrm{P}_{50}\right) \quad$ is profit vector, $\mathrm{X}=\left(\mathrm{X}_{1}, \mathrm{X}_{2}, \cdots, \mathrm{X}_{50}\right)$ is integer set. If $\mathrm{X}_{\mathrm{i}}$ is 1 then produce the product. If $\mathrm{X}_{\mathrm{i}}$ is 0 then not produce the product. $\mathrm{R}=\left(\mathrm{R}_{1}, \mathrm{R}_{2}, \cdots, \mathrm{R}_{50}\right)$ is material vector.

\section{Build and solve procedure of hybrid genetic algorithms}

The solving method of bag problem is some heuristic algorithms mainly (such as greedy algorithms ${ }^{[4]}$ ), we also can use genetic algorithms to solve the problem. We need search $2^{\mathrm{n}}$ points if we use enumerate method aim at bag problem of $n$ goods. We can get approximate optimization result by greedy algorithms, and can not sure get optimization. Simple genetic algorithms can't get good result by genetic algorithms if the scale of the problem is big, and even get worse result than greedy algorithms under many conditions. The reason of the problem is that search space is too big. So we combine heuristic algorithms with greedy algorithms by importing the decode procedure of genetic algorithms, and build one hybrid genetic algorithms.

The composing of hybrid genetic algorithms as follows:

(1)Chromosome code method, we use binary to code vector $\mathrm{X}$ random. It express that we produce the product if $\mathrm{X}_{\mathrm{i}}$ is 1 , and express that we not produce if $\mathrm{X}_{\mathrm{i}}$ is 0 .

(2)Combine of greedy algorithms and decode procedure, above chromosome code method is intuitionistic, but we can't sure get feasible result for that they not satisfy restrict condition aim at random code string, or across and variance unit, that is the code method produce many invalid chromosomes. We can use the idea of greedy algorithms to load the goods that $\mathrm{Pi} / \mathrm{Ri}$ is big and $\mathrm{xi}$ is 1 aim at these chromosome code strings. We change $x i$ into 0 of chromosome code string aim at the goods that should be loaded and should not be loaded in fact. We can produce new and good quality chromosome code string, and they should satisfy restrict condition.

(3)Fit function

$$
f= \begin{cases}\sum_{i=1}^{50} P_{i} X_{i} & \left(\sum_{i=1}^{50} R_{i} X_{i} \leq 5000\right) \\ 0 & \left(\sum_{i=1}^{50} R_{i} X_{i}>5000\right)\end{cases}
$$

(4)Genetic operator, across operation use across operator, variation operation use variation operator, choice operation use choice operator.

We use advanced program language to implement the hybrid genetic algorithms model in this paper, parameter set and run result as follows Figure 2.

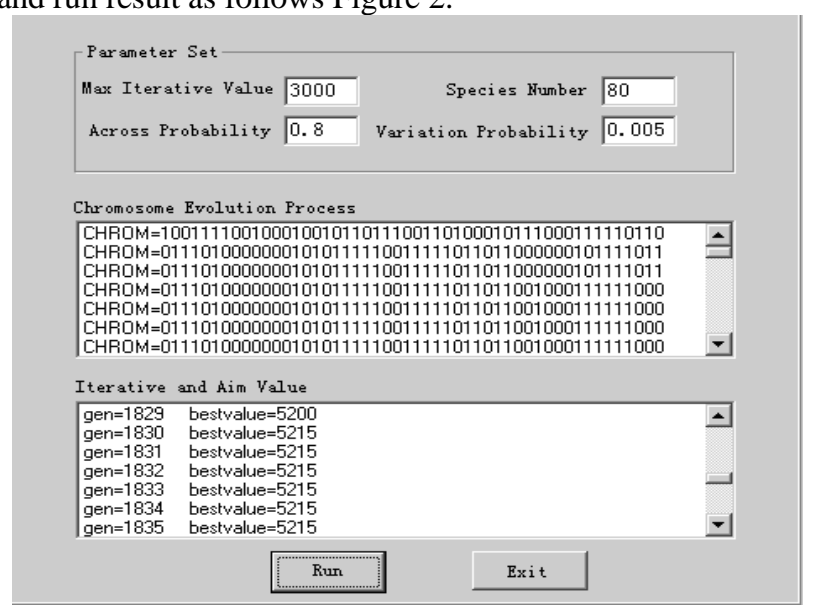

Figure 2 hybrid genetic algorithms parameter and run result

We can get conclusion from picture 2 that the value of chromosome and aim function does not change after iterating 1830. The value of chromosome is how to produce, and the value of aim function is maximal economic benefit.

\section{TAG}

We build one hybrid genetic algorithms model base on genetic algorithms aim at one product optimization problem, 
and realize the model by software. The disposal result of real data tells us that the model is right and has good practicability and accuracy to the problem of product optimization problem. The model has some spread value.

\section{REFERENCES}

[1] Holland J H. Adaptation in Nature and Artificial Systems. MIT Press,1992
[2] De Jong K A. An Analysis of the Behavior of a Class of Genetic Adaptive Systems. Ph. D Dissertation, University of Michigan,No.769381,1975.

[3] Goldberg D E. Genetic Algorithms in Search, Optimization and Machine Learning.Addison-Wesley,1989.

[4] Xie Zheng, Li Jianping. Network Arithmetic and Complexity Theory. Changsha, National Defence Science and Technology University Publishing House, 1995.

[5] Zhou Ming, Sun Shudong. Theory and Application of Genetic Algorithms. Beijing, National Defence Industry Publishing House,2001. P85 P86. 